\title{
A Stochastic Program for Black Start Allocation
}

\author{
Georgios Patsakis \\ Department of Industrial Engineering \\ and Operations Research, \\ Tsinghua-Berkeley Shenzhen Institute, \\ University of California Berkeley \\ gpatsakis@berkeley.edu
}

Abstract

The fast and secure restoration of the power system after an extended blackout highly depends on the location of Black Start (BS) resources. In contrast to most generators, $B S$ units have the ability to start without being connected to an already energized power grid. Selecting a unit to provide BS services is associated with costly technical upgrades, continuous testing, and compensation for the services, and once a unit is selected as $B S$ it is expected to provide that service for several years. For these reasons, the selection process to allocate new $B S$ units is very important and currently handled by experts in the field. Building on the existing literature for power system restoration and black start allocation, we formulate an optimization problem aimed at allocating $B S$ units optimally in the power grid. While restoration plans are usually examined under the assumption of a total blackout, in reality most blackouts are partial, leaving parts of the grid energized and certain elements damaged. In order to account for these cases during the selection process, we formulate a two-stage stochastic program that optimizes the allocation of $B S$ resources over a number of outage scenarios. We use a scenario decomposition algorithm to solve the resulting optimization problem to near-optimality in a high performance computing environment. We conduct numerical experiments using the proposed model and decomposition method on the IEEE-39 test system.

\section{Nomenclature}

\section{Sets}

E Set of branches (ordered pair of buses).

$G \quad$ Set of generators.

$G(i) \quad$ Set of generators connected to bus $i \in N$.

$N \quad$ Set of buses.

\author{
Deepak Rajan \\ Lawrence Livermore \\ National Laboratory \\ rajan3@1lnl.gov
}

$S \quad$ Set of scenarios.

$T \quad$ Set of consecutive integer time instances, starting from 1.

\section{Variables}

$p_{S H_{i}}^{t, s} \quad$ Active power load shed at bus $i \in N$, time $t \in$ $T$ and scenario $s \in S$.

$q_{\text {sys }}^{t, s} \quad$ System-wide reactive power capability at time $t \in T$ for scenario $s \in S$.

$\delta_{i}^{t, s} \quad$ Voltage phase of bus $i \in N$ at time $t \in T$ for scenario $s \in S$.

$f_{g}^{t, s} \quad$ Network flow for energizing paths from generator $g \in G$ at time $t \in T$ for scenario $s \in S$.

$f_{i j}^{t, s} \quad$ Network flow for energizing paths for branch $(i j) \in E$ at time $t \in T$ for scenario $s \in S$.

$p_{g}^{t, s} \quad$ Active power generation of generator $g \in G$ at time $t \in T \cup\{0\}$ for scenario $s \in S$.

$p_{i j}^{t, s} \quad$ Active power flow of branch $(i j) \in E$ at time $t \in T$ for scenario $s \in S$.

$u_{g}^{t, s} \quad$ Binary variable indicating generator $g \in G$ energized at time $t \in T$ for scenario $s \in S$.

$u_{i}^{t, s} \quad$ Binary variable indicating node $i \in N$ energized at time $t \in T$ for scenario $s \in S$.

$u_{B S_{g}} \quad$ Binary variable indicating generator $g \in G$ is BS generator.

$u_{i j}^{t, s} \quad$ Binary variable indicating branch $(i j) \in E$ energized at time $t \in T$ for scenario $s \in S$.

\section{Parameters}

$\cos \left(\phi_{D_{i}}\right)$ Power factor of load at node $i \in N$.

$\epsilon \quad$ Decomposition algorithm termination gap.

$\lambda \quad$ Trade-off coefficient for reactive capability.

$\mu \quad$ Trade-off coefficient for inertia.

$\bar{S}_{i j} \quad$ Maximum flow limit for branch $(i j) \in E$. 
$\pi_{s} \quad$ Weight assigned to scenario $s \in S$.

$\underline{\delta}, \bar{\delta} \quad$ Lower and upper bounds for voltage phases.

$\underline{Q}_{g} \quad$ Minimum reactive power generation from generator $g \in G$.

$\underline{Q}_{S H_{i}} \quad$ Shunt reactor for bus $i \in N$.

$\underline{V}, \bar{V} \quad$ Lower and upper bounds for voltage magnitude.

$B \quad$ Total budget for BS generator installations.

$b_{i j} \quad$ Susceptance for branch $(i j) \in E$.

$B_{S H_{i j}} \quad$ Shunt susceptance of branch $(i j) \in E$.

$C_{g} \quad$ Operational cost of generator $g \in G$.

$C_{B S_{g}} \quad$ Cost of turning $g \in G$ to a BS generator.

$C_{i} \quad$ Cost of load shed in node $i \in N$ after the blackout.

$J_{g} \quad$ Inertia of generator $g \in G$.

$K_{R_{g}} \quad$ Ramp rate of generator $g \in G$.

$P_{g}^{\max } \quad$ Maximum active power generation from generator $g \in G$.

$P_{C R_{g}} \quad$ Cranking power required to be provided to generator $g \in G$ to initiate its start-up.

$P_{D_{i}} \quad$ Available load at bus $i \in N$.

$T_{C R_{g}} \quad$ Time between generator $g \in G$ being energized until it can increase its active power from zero.

$u_{g}^{0, s} \quad$ Binary parameter indicating state of generator $g \in G$ at time $t \in T$ for scenario $s \in S$.

$u_{g}^{\text {avail,s }}$ Binary parameter indicating the availability of generator $g \in G$ for scenario $s \in S$.

$u_{i}^{0, s} \quad$ Binary parameter indicating state of node $i \in N$ at time $t \in T$ for scenario $s \in S$.

$u_{i}^{\text {avail,s }}$ Binary parameter indicating the availability of node $i \in N$ for scenario $s \in S$.

$u_{i j}^{\text {avail,s }}$ Binary parameter indicating the availability of branch $(i j) \in E$ for scenario $s \in S$.

\section{Introduction}

Despite ongoing efforts to increase the reliability of power systems, natural events, human or equipment faults, attacks or other possible causes can still result in large-scale outages [1]. Power System Restoration (PSR), i.e. restoring the grid to normal operation after an outage, is considered a primary objective within the scope of achieving grid resiliency. One of the main challenges is that most of the generators are unable to start without receiving an initial amount of power (cranking power, corresponding to ancillary equipment and initial energy needs) from the power systemfor a certain amount of time (cranking time). The restoration process relies, then, on selected units (called black start units) that have the capability to start on their own. This capability can be achieved through technical upgrades, such as installing a small diesel generator that can provide the initial cranking power to the unit. System operators are often responsible for compensating these units for the black start (BS) service availability, as well as for regular testing of the technical requirements.

For most systems, detailed procedures exist and regular training of the personnel is in place to ensure a quick and efficient response to a possible blackout. These procedures are specific to each power system and they describe the order in which to energize branches and crank generators, aiming to that critical loads will be energized as soon as possible and that the grid will be restored in a secure way. Critical loads include the auxiliary equipment of nuclear power plants, critical natural gas infrastructure, critical communication equipment, or command and control facilities [2]. The restoration process plan is usually devised for the case of a complete blackout but, with the same priorities in mind, other plans can be constructed for cases of partial blackouts.

A number of approaches have been suggested in literature to construct a restoration plan given the location of the BS units. In $[3,4]$, the authors develop a tool that suggests the next step in a restoration sequence. In $[5,6]$ the authors consider instead a mixed integer program (MIP) where binary decision variables correspond to energization steps. A different modeling approach including reactive power considerations is adopted in [7], aiming to motivate the use of microgrids for PSR. A mixed integer non-linear program is formulated in [8] and feasible solutions are found using ant colony optimization. The sectionalization problem is solved in [9] using binary decision diagrams. Including wind power in restoration is discussed in [10]. Literature reviews of relevant approaches are provided in $[11,12]$.

The effectiveness of a restoration plan highly depends on the choice of the black start units in the grid. Some units are inherently more suitable for the role of black start compared to others. For example, pumpedstorage hydro-power plants are ideal to act as black starts, due to the negligible amount of cranking power and cranking time they require and their high ramping capabilities. On the other hand, some units may be better placed within the power grid, i.e. closer to the critical loads. The problem of allocating black start capabilities 
has also been discussed in the literature. General guidelines to heuristically select black start units are available [2,13-15]. In [16, 17], a minimum procurement cost BS allocation problem is formulated. In [18], the BS allocation problem is formulated including an increased detail of the resulting restoration process and solved to near optimality through a heuristic that proposes candidate feasible restoration sequences. All of the aforementioned studies examine the case of a total blackout for the BS allocation problem.

Solving the BS allocation problem based on the scenario of a total blackout, while useful, is not necessarily representative of reality. We rarely expect a complete system outage, rather smaller outages that leave a number of stable islands with functional generators, from where restoration actions can start as well. Also, some parts of the grid may be more prone to outages than others, due to abnormal weather conditions, unpredictable demand or even the local grid configuration. Furthermore, after an outage, we can not expect that all system components will be in their pre-outage condition. Some generators, lines or buses may have suffered faults or permanent damage, which will make them unavailable for the purposes of restoration.

In this work we seek to address the aforementioned challenges by proposing a stochastic program for BS allocation. A number of scenarios is considered, that corresponds to possible partial system outages, as well as possible unavailability of some lines, generators or buses. The black start allocation is optimized over the scenarios (first stage variables), while a different restoration sequence for each scenario is calculated (second stage variables). The resulting MIP can become very large as the number of scenarios increases. In order to achieve tractability, we observe that, since the critical loads and the characteristics of the generators are the same for all scenarios, the allocation found by considering a scenario in isolation could perform well for the other scenarios. A scenario decomposition technique devised to exploit this observation $[19,20]$ is employed to solve the stochastic program. The computational performance of the decomposition technique is illustrated using the IEEE-39 test power system.

The remainder of this paper is organized as follows. Section 2 presents the problem formulation, section 3 describes the decomposition algorithm, section 4 the simulation results and section 5 concludes the paper.

\section{Optimization Model}

In this section we describe the optimization model employed for the BS allocation problem. A two-stage stochastic program describes decisions that happen be- fore uncertainty is revealed, i.e. first stage decisions, and after uncertainty realized, i.e. second stage decisions. For our problem, the first stage decision is the allocation of the black start capabilities to units. This decision is the same for all the scenarios considered, since we make this decision before the occurrence of any outage. Each scenario corresponds to possible partial or total outages of the power grid, as well as the possible unavailability of grid components (lines, generators or buses). Finally, the second stage decisions are the restoration steps that need to be implemented given the scenario that has occurred and based on the BS allocation of the first stage.

\subsection{First Stage}

A binary variable $u_{B S_{g}}$ is associated with the allocation of each unit $g \in G$ as a BS generator (a value of 1 indicates that a unit is allocated). Units can be excluded from being allocated by explicitly setting the variable equal to 0 in the optimization problem. Furthermore, a unit that is already a black start can have its corresponding binary variable preset to 1 (and this variable can be excluded from the budget constraint). The allocation of a unit translates, for our model, to the installation of a diesel generator that will provide the initial cranking power needed by the unit to start.

2.1.1. Budget Constraint Allocating a black start unit is associated with a number of costs [21]. These may include compensation to the utility owner for the service, costs for technical upgrades and costs to regularly test and maintain the equipment. The cost highly depends on the type of the unit and the commitment approach for black starts that the operator adopts. For our model, we assume that all the costs are reduced to a lump sum payment $C_{B S_{g}}$ for unit $g \in G$. Therefore, the following budget constraint is imposed at the first stage. Note that, there might be a black start allocation that achieves a feasible (worse) restoration plan and uses up a smaller installation budget, but in this paper we do not address this trade-off.

$$
\sum_{g \in G} C_{B S_{g}} u_{B S_{g}} \leq B
$$

\subsection{Second Stage}

While the first stage variables (black start allocation variables) are the same for all scenarios, the second stage variables are optimized for every scenario independently, i.e. they are chosen given a known uncertainty realization, which is why there exists a copy of 
these variables for every scenario (i.e. they are all indexed by scenario $s \in S$ ). The scenarios of the model represent different outage cases and possible unavailability of components. Second stage variables are the decisions to be made in order to restore normal operation of the system for each scenario, for a finite time horizon $T$. Among these, there are binary variables that correspond to the energization of buses $u_{i}^{t, s}$, lines $u_{i j}^{t, s}$ and generators $u_{g}^{t, s}$, which become 1 at the time step $t \in T$ if the component is energized.

2.2.1. Scenarios After the blackout, the operator needs to identify the surviving parts of the grid. These are usually stable islands with generation supporting them. The identification process will also determine which components of the grid are inoperable after the outage (due to a severe fault or malfunction), which cannot be used during the restoration process. In our model, the binary parameters $u_{g}^{0, s}$ and $u_{i}^{0, s}$ determine the initially energized grid for scenario $s \in S$ (1 if energized). The parameters $u_{i}^{\text {avail, } s}, u_{i j}^{\text {avail,s }}$ and $u_{g}^{\text {avail, }, s}$ are set to 0 if the corresponding bus, line or generator is unavailable in scenario $s \in S$. Note that in this model, unavailability is constant across all time steps for each scenario. However, a straightforward extension would be to index the parameter by time in order to indicate that a component is available after some reparation/replacement time. This modification also allows to model switches that require manual operation (i.e. they can not be operated using remote control systems, but instead manned units need to be dispatched on-site to operate them), which need a certain amount of time before operation becomes possible. Finally, the scenario generation process for our purposes is synthetic because the simulations employ artificially constructed IEEE test systems. In a real setup, however, the scenarios can be constructed by system experts or historical outage data, based on the individual characteristics of a power system.

2.2.2. Node Active Power Balance The node balancing constraint at every time instant is:

$$
\begin{aligned}
& \sum_{g \in G(i)}\left(p_{g}^{t, s}+P_{C R_{g}}\left(u_{g}^{\text {avail }, s} u_{B S_{g}}-u_{g}^{t, s}\right)\right)+\sum_{j:(j i) \in E} p_{j i}^{t, s} \\
& -\sum_{j:(i j) \in E} p_{i j}^{t, s}=P_{D_{i}}-p_{S H_{i}}^{t, s}, \forall i \in N, \forall t \in T, \forall s \in S .
\end{aligned}
$$

Constraint (2) stipulates the active power conservation at node $i \in N$ for every time instant $t \in T$. Note the following: if a generator is chosen to be $\mathrm{BS}\left(u_{B S_{q}}=1\right)$, then its cranking power is provided for (by an external source), so it can be immediately energized $\left(u_{g}^{1, s}=1\right)$. On the other hand, if we want a non-BS generator $g \in G$ to get energized, the constraint above introduces a negative term $-P_{C R_{g}}$, so the cranking power needs to be provided for either by a different generator in the same node or by incoming power flows. In the initial phases of the restoration, this constraint will ensure that only the generators that are assigned to be BS or are already connected to an energized island can be energized.

Usually, when a load is picked up after an extended outage, the demand is greater than before the outage. This phenomenon is referred to as cold load pickup. Some of the factors that affect the magnitude and duration of cold load are outage duration, type of load, time of day and load level. One reason for this phenomenon is that, while some loads are usually diverse and cycle, after the re-energization they tend to all draw current at the same time for several minutes [22]. Despite the uncertainty in the load when closing the switches, the operators usually have the ability to pick load in small enough chunks. Even more, load is used as a tool to alleviate overvoltages and increase the system stability (by allowing more generation to be committed as well). For these reasons, load behaves more like a decision variable for restoration purposes. Therefore, in our model, a continuous load shed variable is employed, that satisfies:

$$
\begin{gathered}
\left(1-u_{i}^{t, s}\right) P_{D_{i}} \leq p_{S H_{i}}^{t, s} \leq P_{D_{i}}, \\
\forall i \in N, \forall t \in T, \forall s \in S .
\end{gathered}
$$

2.2.3. Reactive Power Reactive power capability is important in maintaining the voltages of the power system within security limits. For this model, we introduce a system-wide reactive power capability variable $q_{\text {syst }}^{t, s}$.

$$
\begin{gathered}
\sum_{i \in N} \sum_{g \in G(i)} \underline{Q}_{g} u_{g}^{\max \left\{0, t-T_{C R_{g}}-1\right\}, s}+\sum_{(i j) \in E} B_{S H_{i j}} u_{i j}^{t, s} \\
+\sum_{i \in N} Q_{S H i} u_{i}^{t, s}-\sum_{i \in N}\left(P_{D_{i}}-p_{S H_{i}}^{t, s}\right) \tan \left(\phi_{D_{i}}\right)=q_{\mathrm{syst}}^{t, s}, \\
\forall t \in T, \forall s \in S .
\end{gathered}
$$

A line injects reactive power $\frac{1}{2} B_{S H_{i j}} V^{2} u_{i j}^{t}$ at each of the buses it connects to, if energized, where the bus voltage $V$ is assumed close to 1.0pu for this constraint, in order to allow for a linear formulation. The reactive power can be absorbed by either generators that have been energized at least $T_{C R_{g}}+1$ time units in advance, by reactive compensation connected to the bus $Q_{S H_{i}}$, or by 


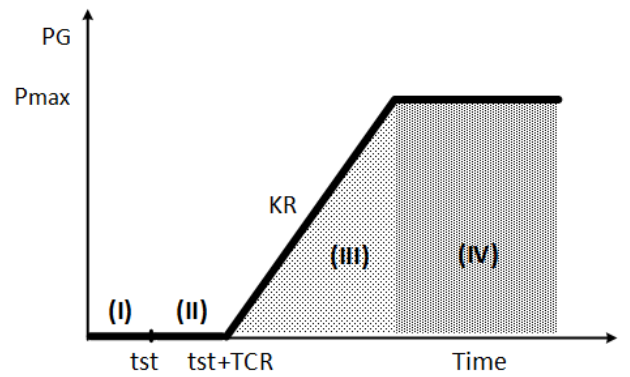

(a) Generator power output. A generator that gets energized at time $t_{s t}$ needs to be cranked for $T_{C R}$ periods before it can inject power to the grid. $T_{C R}+1$ periods after energized, the generator can ramp up its active power production with a maximum rate of $K_{R}$, until its maximum generation limit $P_{\max }$ is reached.

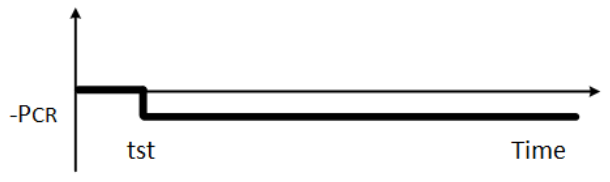

(b) Cranking power of generator unit. As soon as the generator is energized $\left(u_{g}^{t, s}=1\right)$, it needs to absorb power $P_{C R}$. This power is either provided by other generators (if the unit does not have BS capability), or by a dedicated diesel BS unit or battery (if the unit has BS capabilities)

Figure 1: Typical generator curve. The parameters $T_{C R}, P_{C R}, T_{C R}$, $P_{\max }$ vary depending on the type of generator.

loads with lagging power factor $\left(\tan \left(\phi_{D_{i}}\right)>0\right)$. The load is assumed picked up at a constant power factor, as in [23] and [7]. The modeling of the system-wide reactive power follows the ideas in [6].

2.2.4. Generator Model A typical generator startup model is assumed, following similar assumptions as in [5, 18]. Fig. 1 depicts these assumptions. The binary variable $u_{g}^{s, t}$ is associated with the energization status of generator $g \in G$. This variable is exogenously defined based on the availability of active power or BS unit assignment in constraint (2). The cranking power requirement corresponds to Fig. 1b. The equations that describe Fig. 1a are:

$$
\begin{aligned}
& 0 \leq p_{g}^{\tau, s} \leq P_{g}^{\max } u_{g}^{t, s}, \forall g \in G, \\
& \forall \tau \in\left\{t, t+1, \ldots, t+T_{C R_{g}}+1\right\}, \forall t \in T \cup\{0\}, \forall s \in S,
\end{aligned}
$$

$p_{g}^{t, s}-p_{g}^{t-1, s} \leq K_{R_{g}}, \forall g \in G, \forall t \in T, \forall s \in S$

$p_{g}^{t-1, s}-p_{g}^{t, s} \leq K_{R_{g}}, \forall g \in G, \forall t \in T, \forall s \in S$
Constraint (5a) makes sure that the active power cannot be positive for at least $T_{C R_{g}}$ units of time after the generator is energized, both for BS and for non BS generators. Also, the maximum active power limit is imposed at all time instances that the generator has positive active power production. The ramping rate capability is imposed through constraints (5b) and (5c).

2.2.5. Line Switching A constraint that a line can have nonzero flow only if it has been switched on by the restoration process needs to be imposed. For that purpose, the transmission switching modeling with the dc approximation is utilized [24]. The constraints that impose this requirement are:

$$
-\bar{S}_{i j} u_{i j}^{s, t} \leq p_{i j}^{t, s} \leq u_{i j}^{t, s} \bar{S}_{i j}, \forall(i j) \in E, \forall t \in T, \forall s \in S,
$$

$\underline{\delta} \leq \delta_{i}^{t, s} \leq \bar{\delta}, \forall i \in N, \forall t \in T, \forall s \in S$

Constraint (6a) is linearized using the big-M reformulation:

$$
\begin{array}{r}
b_{i j}\left(\delta_{i}^{t, s}-\delta_{j}^{t, s}\right)-p_{i j}^{t, s}+\left(1-u_{i j}^{t, s}\right) M_{i j} \geq 0, \\
\forall(i j) \in E, \forall t \in T, \forall s \in S, \\
b_{i j}\left(\delta_{i}^{t, s}-\delta_{j}^{t, s}\right)-p_{i j}^{t, s}-\left(1-u_{i j}^{t, s}\right) M_{i j} \leq 0, \\
\forall(i j) \in E, \forall t \in T, \forall s \in S,
\end{array}
$$

where $M_{i j} \geq\left|b_{i j}\right|(\bar{\delta}-\underline{\delta})$. Let us note here that the dc approximation of the power flow equations together with the aggregate reactive power constraint are not an accurate representation of the system. However, for the purposes of the BS allocation problem, this simplified approach that still retains the main characteristics of a complete model is adopted in order to achieve tractability.An increased accuracy can be obtained (especially for the cases where the optimization problem is aiming to identify a restoration sequence) by using the ac power flow equations or dedicated ac approximations of the power flow equations, such as the one in [25].

\subsection{Consistency of energized grid}

A series of constraints that ensure the consistency of the grid are imposed. By consistency, we mean that any island of the grid needs to have at least one energized generator to support it. Equivalently, we need to ensure that for any energized component of the grid (line or bus), there exists a path of energized lines that lead to a node with an energized generator. One established way to impose that is the following set of constraints, that 
make use of network flow variables:

$$
\begin{aligned}
& 0 \leq f_{g}^{t, s} \leq u_{g}^{t, s}, \forall g \in G, \forall t \in T, \forall s \in S, \\
& -u_{i j}^{t, s} \leq f_{i j}^{t, s} \leq u_{i j}^{t, s}, \forall(i j) \in E, \forall t \in T, \forall s \in S, \\
& \sum_{j:(j i) \in E} f_{j i}^{t, s}-\sum_{j:(i j) \in E} f_{i j}^{t, s}+\sum_{g \in G(i)} f_{g}^{t, s}=\frac{1}{N} u_{i}^{t, s}, \\
& \forall i \in N, \forall t \in T, \forall s \in S .
\end{aligned}
$$

Constraints (8a), (8b) and (8c) impose a feasibility problem given fixed values of $u_{g}^{t, s}, u_{i j}^{t, s}$ and $u_{i}^{t, s}$ for the flows $f_{i j}^{t, s}$ and $f_{i}^{t, s}$. A node can be energized $\left(u_{i}^{t, s}=1\right)$ if there is a feasible flow from one or more of the generators with $u_{g}^{t, s}=1$, flowing only through branches with $u_{i j}^{t, s}=1$, such that the load of that node $\frac{1}{N} u_{i}^{t, s}$ can be satisfied. Otherwise, the state of that node has to be $u_{i}^{t, s}=0$. We also impose the following constraints:

$$
u_{i j}^{t, s} \leq u_{i}^{t, s}, u_{i j}^{t, s} \leq u_{j}^{t, s}, \forall(i j) \in E, \forall t \in T, \forall s \in S,
$$

i.e. a branch cannot be energized unless both of the nodes connected to it are energized. Also, if any generator connected to a node is energized, then the node is considered energized:

$$
u_{g}^{t, s} \leq u_{i}^{t, s}, \forall i \in N, \forall g \in G(i), \forall t \in T, \forall s \in S
$$

We include a time staging constraint which imposes that a line can only be energized at time $t$ if one of its nodes was energized at time $t-1$.

$$
u_{i j}^{t, s} \leq u_{i}^{t-1, s}+u_{j}^{t-1, s}, \forall(i j) \in E, \forall t \in T, \forall s \in S .
$$

Finally, we assume that buses and generators, once energized, must remain energized until the end of the horizon:

$$
\begin{aligned}
& u_{g}^{t, s} \geq u_{g}^{t-1, s}, \forall g \in G, \forall t \in T, \forall s \in S, \\
& u_{i}^{t, s} \geq u_{i}^{t-1, s}, \forall i \in N, \forall t \in T, \forall s \in S .
\end{aligned}
$$

2.3.1. Component unavailability We model the possible unavailability of components in a scenario using the parameters $u_{g}^{\text {avail,s }}$ for generators, $u_{i}^{\text {avail, } s}$ for nodes, and $u_{i j}^{\text {avail,s }}$ for lines, which are equal to 1 if the corresponding component is available and 0 otherwise. The following constraints are added to the formulation to ensure that an unavailable component will not be used or energized throughout the restoration process:

$$
\begin{aligned}
& u_{g}^{t, s} \leq u_{g}^{\text {avail }, s}, \forall g \in G, \forall t \in T, \forall s \in S, \\
& u_{i}^{t, s} \leq u_{i}^{\text {avail }, s}, \forall i \in N, \forall t \in T, \forall s \in S, \\
& u_{i j}^{t, s} \leq u_{i j}^{\text {avail }, s}, \forall i \in N, \forall t \in T, \forall s \in S .
\end{aligned}
$$

\subsection{Objective function}

The objective of the problem highly depends on the specific power system we are interested in. A generic form of objective, that is also used in this paper, can be stated as follows:

$$
\begin{aligned}
& \text { minimize } \sum_{s \in S} \pi_{s}\left(\sum_{t \in T} \sum_{i \in N} C_{i}^{t} p_{S H_{i}}^{t, s}\right. \\
&\left.+\lambda \sum_{t \in T} q_{\mathrm{sys}}^{t, s}-\mu \sum_{t \in T} \sum_{g \in G} u_{g}^{t, s} J_{g}\right)
\end{aligned}
$$

The objective penalizes: (i) the load shed (depending on how critical the load that is being shed is at various time instances after the blackout), (ii) the reactive power capacity (a negative reactive power capacity ensures that the reactive power injected by the high voltage transmission lines during the low load operating points of restoration can be absorbed), and (iii) the additive inverse of the total inertia of the system (higher inertia leads to higher system stability).

\section{Scenario Decomposition Approach}

The size of the stochastic program grows linearly with the number of scenarios, since a copy of the second stage variables is added for every scenario, along with the corresponding constraints. Even though there are techniques to reduce the number of scenarios [26] or carefully select them, the number of scenarios necessary for the needs of a problem can be large, especially when the underlying uncertainty is characterized by low-probability high-impact events (such as component unavailability). For this reason, special purpose algorithms have been developed to decompose the problem by scenario. These algorithms aim to solve smaller optimization problems corresponding to one or more scenarios (which may be easier to solve) and then combine the information to approach the solution of the complete stochastic program. In this section, we describe the decomposition algorithm of $[19,20]$ in the context of our problem.

Let $\boldsymbol{u}_{B S} \in \mathbb{B}^{|G|}$ be the vector of the first stage BS allocation, and $\boldsymbol{y}_{s}$ be a vector that contains all the second stage variables for scenario $s \in S$. Let $X$ be the 
feasibility set imposed by the constraints involving only first stage variables:

$$
X=\left\{\boldsymbol{u}_{B S} \in \mathbb{B}^{|G|}: \sum_{g \in G} C_{B S_{g}} u_{B S_{g}} \leq B\right\} .
$$

Let $Y_{s}\left(\boldsymbol{u}_{B S}\right)$ be the set to which $\boldsymbol{y}_{s}$ must belong, enforced by the rest of the constraints (including the integrality of the energization variables), for scenario $s \in S$, if the first stage variables are fixed at a value of $\boldsymbol{u}_{B S}$ :

$$
Y_{s}\left(\boldsymbol{u}_{B S}\right)=\left\{\boldsymbol{y}_{s}:(2)-(5),(7)-(13)\right\}, \forall s \in S .
$$

Finally, define the functions $f_{s}$, for $s \in S$, that return the optimal value of the second stage optimization problem for scenario $s$ given the BS allocation $\boldsymbol{u}_{B S}$ :

$$
\begin{array}{rr}
f_{s}\left(\boldsymbol{u}_{B S}\right)={\underset{\boldsymbol{y}}{s} \in Y_{s}\left(\boldsymbol{u}_{B S}\right)}_{t \in T}^{\operatorname{minimize}} & \sum_{t \in N} \sum_{i}^{t} p_{S H_{i}}^{t, s} \\
\lambda \sum_{t \in T} q_{\mathrm{sys}}^{t, s} & -\mu \sum_{t \in T} \sum_{g \in G} u_{g}^{t, s} J_{g}
\end{array}
$$

Based on these definitions, the stochastic BS allocation problem can be rewritten as:

$$
\underset{\boldsymbol{u}_{B S} \in \boldsymbol{X}}{\operatorname{minimize}} \sum_{s \in S} \pi_{s} f_{s}\left(\boldsymbol{u}_{B S}\right)
$$

The binary nature of the first stage decisions in (18) is what allows the decomposition scheme proposed in [19] and elaborated in [20] to be used. The steps of the algorithm are described in Fig. 2.

The main body of the algorithm is divided into three phases, the Lower Bounding Phase, the Upper Bounding Phase and the Cut Phase. In the Lower Bounding Phase, we fix every scenario $s \in S$ and solve for the optimal first stage decision given that scenario, over a space $X \backslash W$. This yields $|S|$ scenario specific solutions for the first stage variables $\boldsymbol{u}_{B S}$ at iteration $t$. In the first iteration, the set $W$ is empty, so we are essentially solving $|S|$ scenario subproblems without any interaction, i.e. we are solving the initial problem after relaxing the non-anticipativaty constraints. Since we are solving a relaxation, at least for the first iteration, we are guaranteed to get a lower bound on the optimal solution to (18). For the next iterations, we get lower bounds for (18) solved over the restricted space of first stage variables $X \backslash W$.

In the Upper Bounding Phase of the algorithm, the $|S|$ scenario specific solutions for the first stage variables found during the previous phase are tested into the full

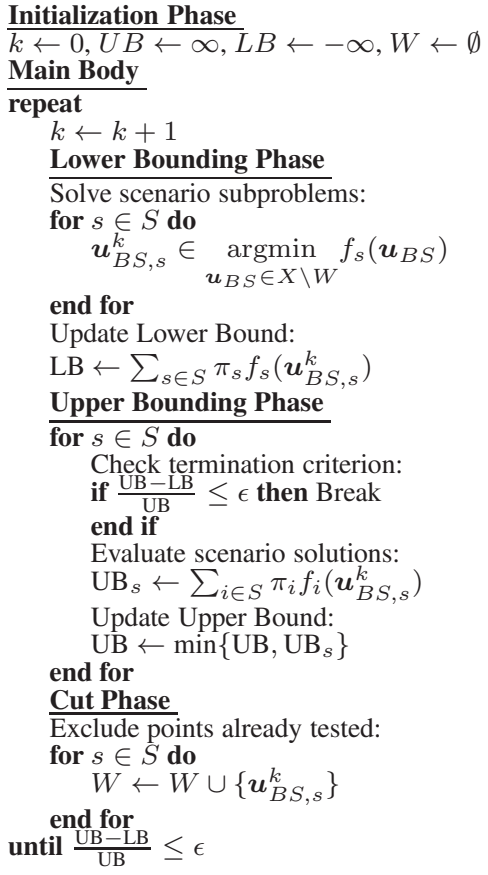

Figure 2: Decomposition scheme from [19] applied to the BS Allocation Problem. The Lower Bounding Phase involves solving smaller optimization problems than the original, since the scenario is fixed, whereas the Upper Bounding Phase involves smaller problems since both the first stage and the scenario are fixed (just evaluations of the function $f_{s}$ ).

problem. If feasible, each one of them yields an upper bound to (18). That way, we can possibly update the upper bound and the first stage solution that yields it.

Finally, in the Cut Phase, we add the points $\left\{\boldsymbol{u}_{B S, s}^{k}\right\}_{s \in S}$ in the set $W$. Our objective function value has already been calculated for all of these points during the previous phase, so we can exclude them from any further consideration. This is achieved by adding a global cut in the optimization problems solved in the first phase, for every point in $W$. The following "NoGood-Cut" is employed to cut off the point $\boldsymbol{u}_{B S, s}^{k}$ :

$$
\boldsymbol{u}_{B S}^{T}\left(\mathbf{1}-\boldsymbol{u}_{B S, s}^{k}\right)+\left(\mathbf{1}-\boldsymbol{u}_{B S}\right)^{T} \boldsymbol{u}_{B S, s}^{k} \geq 1 .
$$

The algorithm will terminate once the desired optimality guarantee $\epsilon$ is achieved. Due to the construction of the algorithm, it is guaranteed to terminate in a finite number of steps (since there are only a finite number of binary points in the space of the first stage variables and each step eliminates at least one). Of course, for suitable problems, the algorithm is expected to terminate much earlier in practice. A setup where this would occur is when the solutions obtained by solving for individual scenarios are close to each other. If the first stage solution for a scenario in the first phase of the algorithm yields a reasonable allocation for other scenar- 
ios as well, that implies that a tight upper bound will be obtained in the second phase of the algorithm. Even more, if the individual scenario first stage solutions are only slightly different from each other, by eliminating them from future consideration in the next iteration of the algorithm, we may end up with individual scenario solutions that are the same for all scenarios. The black start allocation problem is a suitable candidate, since the main driving forces of the allocation of BS units are the location of the critical loads and the characteristics of each generator (a small cranking time and high ramping rate usually make for an ideal BS unit), all of which are the same across scenarios. The differentiation caused by the scenario specific initial stable islands and component unavailability might lead to slightly different allocations for the individual scenarios, which can be eliminated using the "No-Good-Cuts".

\section{Simulation Results}

All the simulations are performed using the Cab cluster of the Lawrence Livermore National Laboratory. Each node of the Cab cluster has two Intel Xeon E52670 processors at $2.6 \mathrm{GHz}$ and $32 \mathrm{~GB}$ of RAM memory. We formulate the mathematical programs using Mosel 4.0.4 and use Xpress 8.5.0 for solving them [27]. The decomposition algorithm was parallelized in 6 nodes with 2 jobs per node (i.e. solving up to 12 mathematical programs in parallel) and 8 threads per job (i.e. setting Xpress to use 8 threads for traversing the branchand-bound tree). A simple recursive function in Python, described in Fig. 3, was used to generate the synthetic scenarios. We use Matlab to manage and visualize the results.

\subsection{Simulation of the IEEE-39 Bus System}

In order to illustrate the effectiveness of the proposed model, a small test case is initially considered. The IEEE-39 bus system consists of 39 buses, 10 generators and 34 branches [28]. The parameters used can be found in [18]. The parameters for generator 10 are purposefully chosen in a way that favors turning it into a BS unit (i.e. small cranking power of $1 \mathrm{MW}$ and a small cranking time of 10 minutes). The length of the time horizon is set to $T=40$ time units, with a $5 \mathrm{~min}$ utes time step, whereas 20 equally probable scenarios are used in the stochastic program. Some of the scenarios are depicted in Fig. 4. The problem has 596541 constraints and 229010 variables, of which 76010 are binary. Without the decomposition algorithm, Xpress is unable to even find a feasible point after 10 hours of execution in a node of the Cab cluster utilizing 16 threads (and default settings).

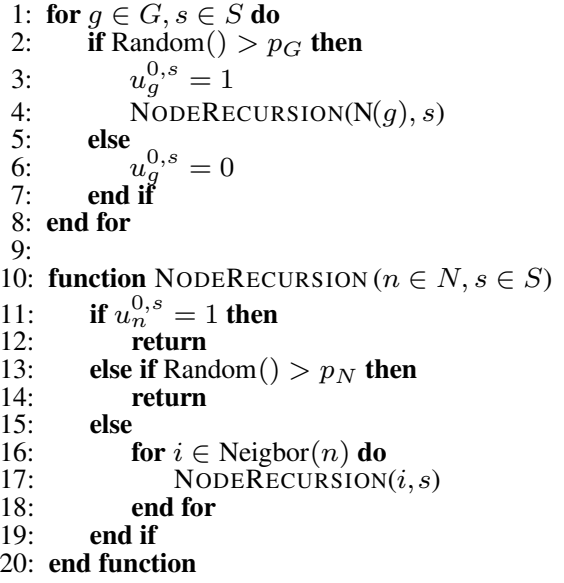

Figure 3: Code that generates the initial islands for every scenario in a way that each island contains (at least) one energized (isochronous) generator. Note that $\mathrm{N}(g)$ is the node to which generator $g \in G$ is connected, Neighbor $(i)$ is the set of neighboring nodes to node $i \in$ $N$ and Random is a (different for every call) uniformly distributed random variable in $[0,1]$. As far as the component unavailability is concerned, every initially de-energized component in a scenario was considered unavailable for the whole process with probability 0.001 .

\begin{tabular}{|l|c|}
\hline Number of scenarios & 20 \\
\hline Variables per scenario & 11450 \\
\hline Constraints per scenario & 29437 \\
\hline Binaries per Scenario & 3202 \\
\hline $\begin{array}{l}\text { Lower Bounding Phase Mean } \\
\text { Time [s] }\end{array}$ & 292 \\
\hline $\begin{array}{l}\text { Upper Bounding Phase Mean } \\
\text { Time [s] }\end{array}$ & 118 \\
\hline $\begin{array}{l}\text { Mean Time for solution evalua- } \\
\text { tion [s] }\end{array}$ & 126 \\
\hline Total Algorithm Time [s] & 6700 \\
\hline
\end{tabular}

Table 1: Computational performance of the decomposition algorithm. Note that not all solution evaluations need to happen at the second stage, since some of the solutions found by the subproblems of the Lower Bounding Phase are repeated and the evaluations in the Upper Bounding Phase for the repeated points happen only once.

The convergence behavior of the algorithm can be seen in Fig. 5. A computational study of the scenario decomposition algorithm is presented in Table 1. The algorithm terminates after 5 iterations. The solution yields the allocation of two black start units, at generators 6 and 10. The initial restoration steps are depicted in Fig. 6. The total generation and total load for two scenarios are shown in Fig. 7.

\section{Conclusions}

We presented a model for the problem of optimal BS allocation for power system restoration in the form of a two-stage stochastic program. The model also captures the basic characteristics of the restoration process. Different scenarios corresponding to different initial states of the grid failure and different component availability for the restoration process are considered and a scenario 

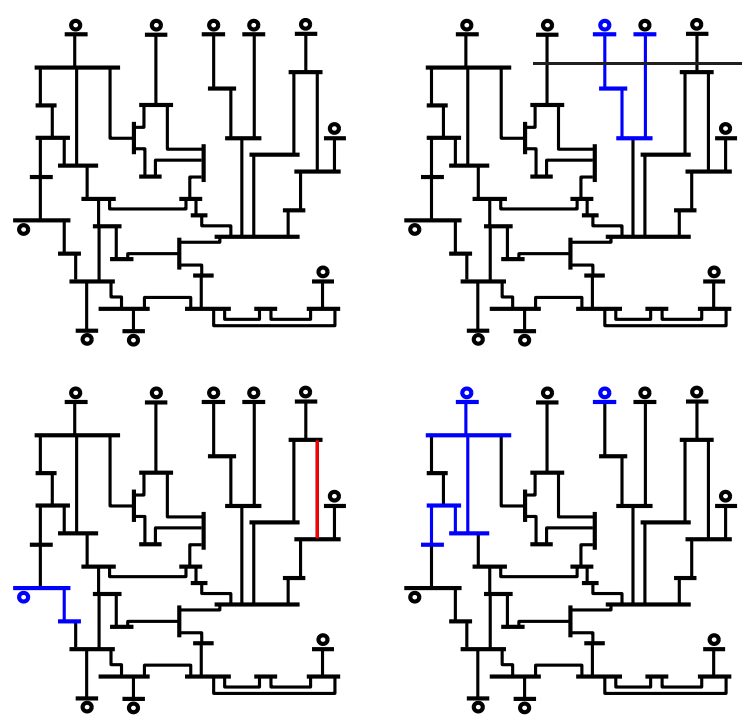

Figure 4: A few of the different scenarios considered in the simulations. Components in black indicate de-energized parts of the system, components in blue indicate the initial stable islands and components in red indicate unavailability. Initial line variables are not employed in our model, but we assume that initially a line between two energized nodes is energized. The top left scenario is the case of a total blackout. The top right and bottom left scenarios have one initial energized island each, but the bottom left scenario has one line that is unavailable for the whole restoration process. The bottom right scenario has two initial islands, each one with a functional generator.

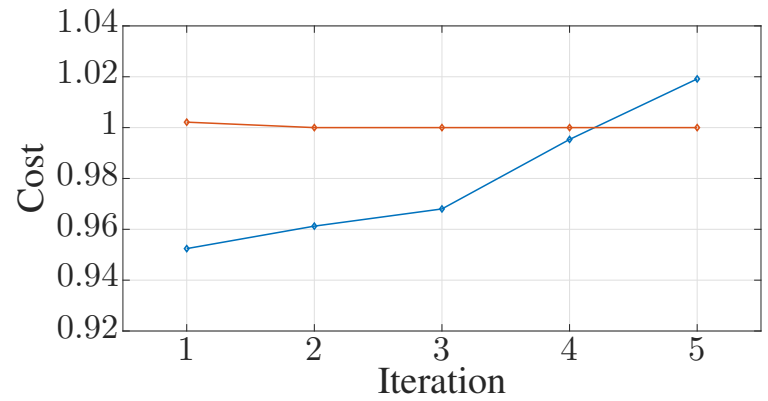

Figure 5: Convergence behavior of the decomposition algorithm in Fig. 2, for the IEEE-39 bus system. The UB (red line) is decreasing and the LB (blue line) increasing. Note that, since during the LB evaluation feasible solutions are chopped off by the No-Good-Cuts, the LB is not necessarily a lower bound of the stochastic problem. However, when LB becomes higher than the running UB, we have a guarantee that a near optimal solution (within the precision that the upper bounding phase subproblems are solved) is found (corresponding to the current UB).
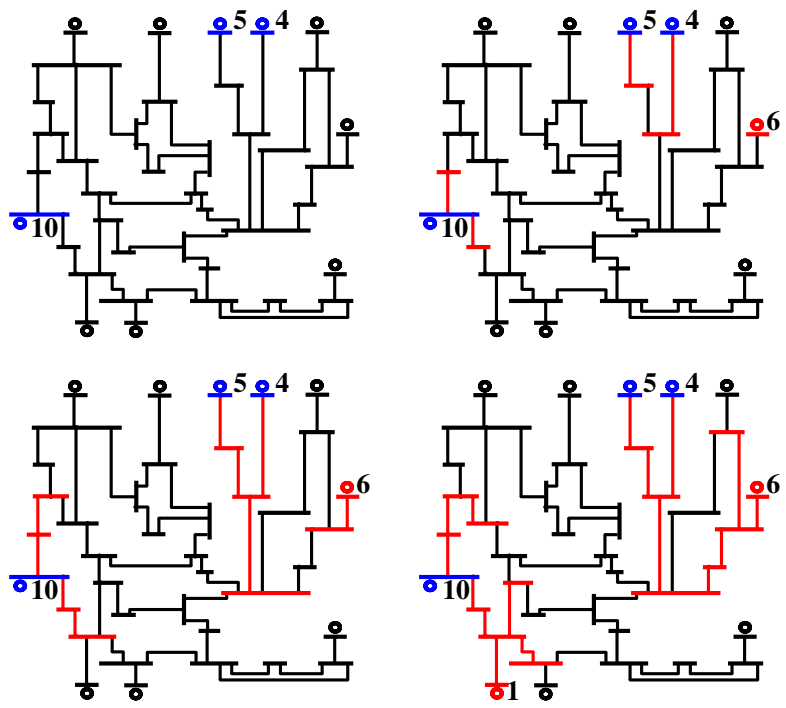

Figure 6: Initial restoration steps for the IEEE-39 bus system in a scenario where generators 4,5 and 10 are initially energized (pictured in blue in the upper left figure). Generator 6 is a BS unit, so it can start at time step 1. Generator 10 is also a BS unit, but it was not influenced by the blackout, so it did not have to restart. The restoration steps (around the initial stable islands and the BS unit) can be seen in red.

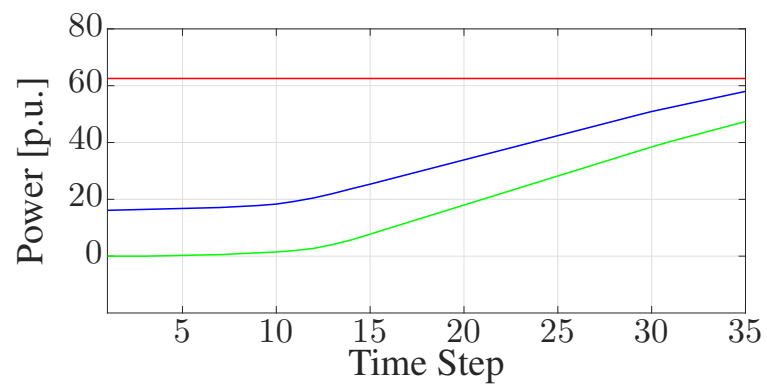

Figure 7: Plot of total system load (red line) with the total generation for the scenario of total blackout (green line) and a scenario with two initially energized generators (blue line) for the 39 bus system. Note that in the case of a total blackout, the generation power starts from zero and ramps up, while in the other scenario the total generation starts from a positive value, since not all of the grid is out of service. 
decomposition algorithm is tested. Simulations verify the effectiveness of the proposed approach for a test system.

\section{Acknowledgments}

This work was performed under the auspices of the U.S. Department of Energy by Lawrence Livermore National Laboratory under Contract DE-AC5207NA27344. Partial support for this work was also received from The Berkeley Tsinghua Shenzhen in statute and from ARO grant W911NF-17-1-0555. The authors would like to thank FICO for providing licenses for Xpress Optimizer and Gurobi for the licenses to the Gurobi Optimizer. Georgios Patsakis is a Fellow of the Onassis Foundation.

\section{References}

[1] J. Minkel, "The 2003 Northeast Blackout-Five Years Later," Scientific American, vol. 13, 2008.

[2] PJM Manual: System Restoration, PJM, 6 2017, rev. 24.

[3] Y. Hou, C. C. Liu, K. Sun, P. Zhang, S. Liu, and D. Mizumura, "Computation of milestones for decision support during system restoration," IEEE Transactions on Power Systems, vol. 26, no. 3, pp. 1399-1409, Aug 2011.

[4] S. Liu, R. Podmore, and Y. Hou, "System restoration navigator: A decision support tool for system restoration," in Power and Energy Society General Meeting, 2012 IEEE. IEEE, 2012, pp. 1-5.

[5] W. Sun, C.-C. Liu, and L. Zhang, "Optimal generator start-up strategy for bulk power system restoration," IEEE Transactions on Power Systems, vol. 26, no. 3, pp. 1357-1366, 2011.

[6] Y. Jiang, S. Chen, C.-C. Liu, W. Sun, X. Luo, S. Liu, N. Bhatt, S. Uppalapati, and D. Forcum, "Blackstart capability planning for power system restoration," International Journal of Electrical Power \& Energy Systems, vol. 86, pp. 127-137, 2017.

[7] A. Castillo, "Microgrid provision of blackstart in disaster recovery for power system restoration," in Smart Grid Communications, 2013 IEEE International Conference on. IEEE, 2013, pp. 534-539.

[8] Y.-T. Chou, C.-W. Liu, Y.-J. Wang, C.-C. Wu, and C.-C. Lin, "Development of a black start decision supporting system for isolated power systems," IEEE Transactions on Power Systems, vol. 28, no. 3, pp. 2202-2210, 2013.

[9] C. Wang, V. Vittal, and K. Sun, "OBDD-based sectionalizing strategies for parallel power system restoration," IEEE Transactions on Power Systems, vol. 26, no. 3, pp. 1426-1433, 2011.

[10] A. Golshani, W. Sun, Q. Zhou, Q. P. Zheng, and Y. Hou, "Incorporating wind energy in power system restoration planning," IEEE Transactions on Smart Grid, 2017.

[11] L. Yutian, F. Rui, and V. Terzija, "Power system restoration: a literature review from 2006 to 2016," Journal of Modern Power Systems and Clean Energy, vol. 4, no. 3 , pp. 332-341, 2016.

[12] A. Castillo, "Risk analysis and management in power outage and restoration: A literature survey," Electric Power Systems Research, vol. 107, pp. 9-15, 2014.
[13] N. Saraf, K. McIntyre, J. Dumas, and S. Santoso, "The annual black start service selection analysis of ERCOT grid," IEEE Transactions on Power Systems, vol. 24, no. 4, pp. 1867-1874, 2009.

[14] W. Sun, C.-C. Liu, and S. Liu, "Black start capability assessment in power system restoration," in Power and Energy Society General Meeting, 2011 IEEE. IEEE, 2011, pp. 1-7.

[15] R. Kafka, "Review of PJM restoration practices and NERC restoration standards," in Power and Energy Society General Meeting-Conversion and Delivery of Electrical Energy in the 21st Century, 2008 IEEE. IEEE, 2008, pp. 1-5.

[16] F. Qiu, J. Wang, C. Chen, and J. Tong, "Optimal black start resource allocation," IEEE Transactions on Power Systems, vol. 31, no. 3, pp. 2493-2494, 2016.

[17] F. Qiu and P. Li, "An integrated approach for power system restoration planning," Proceedings of the IEEE, 2017.

[18] G. Patsakis, D. Rajan, I. Aravena, J. Rios, and S. Oren, "Optimal black start allocation for power system restoration," IEEE Transactions on Power Systems, 2018.

[19] S. Ahmed, "A scenario decomposition algorithm for 01 stochastic programs," Operations Research Letters, vol. 41, no. 6, pp. 565-569, 2013

[20] K. Ryan, D. Rajan, and S. Ahmed, "Scenario decomposition for 0-1 stochastic programs: Improvements and asynchronous implementation," in Parallel and Distributed Processing Symposium Workshops, 2016 IEEE International. IEEE, 2016, pp. 722-729.

[21] ISO New England, "Schedule 16 - Blackstart standard rate report," https://www. iso-ne.com/isoexpress/web/reports/billing/-/tree/ schedule-16---blackstart-standard-rate-report, 2016, [Online; accessed Aug-2017].

[22] F. Friend, "Cold load pickup issues," in Protective Relay Engineers, 2009 62nd Annual Conference for. IEEE, 2009, pp. 176-187.

[23] C. Coffrin and P. Van Hentenryck, "Transmission system restoration with co-optimization of repairs, load pickups, and generation dispatch," International Journal of Electrical Power \& Energy Systems, vol. 72, pp. 144-154, 2015.

[24] K. W. Hedman, R. P. O’Neill, E. B. Fisher, and S. S. Oren, "Optimal transmission switching with contingency analysis," IEEE Transactions on Power Systems, vol. 24, no. 3, pp. 1577-1586, 2009.

[25] I. Aravena, D. Rajan, and G. Patsakis, "Mixed-integer linear approximations of ac power flow equations for systems under abnormal operating conditions," in Power and Energy Society General Meeting (PES), 2018 IEEE. IEEE.

[26] H. Heitsch and W. Römisch, "A note on scenario reduction for two-stage stochastic programs," Operations Research Letters, vol. 35, no. 6, pp. 731-738, Nov. 2007.

[27] C. Guéret, C. Prins, and M. Sevaux, "Applications of optimization with Xpress-MP," contract, p. 00034, 1999.

[28] T. Athay, R. Podmore, and S. Virmani, "A practical method for the direct analysis of transient stability," IEEE Transactions on Power Apparatus and Systems, no. 2, pp. 573-584, 1979. 